\title{
Dieta e Cárie Dental em Escolares de 10 a 14 Anos na Cidade de Picos, Piauí
}

\section{Diet and Dental Caries in Schoolchildren from 10 to 14 Years Old in the City of Picos, Piauí}

Selma Maria Santos Moura*; Ingrid Macedo de Oliveira ${ }^{\mathrm{b}}$; Carla Maria de Carvalho Leite ${ }^{\mathrm{b}}$; Aírton Mendes Conde Júnior ${ }^{\mathrm{b}}$

aUniversidade Federal do Piauí, Centro de Ciências da Saúde.

bUniversidade Federal do Piauí, Programa de Pós-Graduação Stricto Sensu em Odontologia.

*E-mail: selmoura@gmail.com.

Recebido em: 05/10/2015; Aceito em: 09/11/2015

\begin{abstract}
Resumo
A dieta e o estado nutricional influenciam na saúde geral e bucal dos indivíduos. Alimentos ricos em açúcares, por sua vez, facilitam o desenvolvimento da doença cárie. Conhecer os hábitos alimentares de uma população e a prevalência de cárie auxilia no entendimento da relação entre essas duas condições e, por conseguinte, na criação de medidas preventivas ao desenvolvimento da cárie dentária que leva muitas crianças e adolescentes à perda precoce dos dentes. Dessa forma, buscou-se avaliar a presença de cárie dentária e os hábitos alimentares de escolares de 10 a 14 anos de uma escola municipal no município de Picos, Piauí. Estudo observacional transversal realizado com os 69 alunos regularmente matriculados na faixa de 10 a 14 anos. Foram aplicados questionários que abordaram temas referentes à higiene bucal, utilização de serviços odontológicos e aos hábitos alimentares, com ênfase no consumo de açúcar. Exames clínicos para deteç̧ão de lesões cariosas também foram realizados. A análise descritiva dos dados foi representada por meio de tabelas e gráficos. Em $66,6 \%$ dos indivíduos a cárie estava presente, e 7,4\% deles tinham seis ou mais lesões. Cereais, biscoitos e refrigerantes foram alguns dos alimentos mais consumidos (78,2\%, 60,8\% e 55\% respectivamente) e apenas 44,9\% dos escolares relataram realizar a escovação após lanches. Concluiu-se que, na população em estudo, a maioria dos indivíduos tinha elementos dentários com lesões cariosas e alimentação rica em carboidrato e açúcares.

Palavras-chave: Hábitos Alimentares. Nutrição em Saúde Pública. Cárie Dentária. Higiene Bucal.
\end{abstract}

\begin{abstract}
Diet and nutritional status influence in general and oral health of individuals. Foods high in sugars in turn, facilitate the development of caries. Know the eating habits of a population and the prevalence of caries helps in understanding the relationship between these two conditions and therefore the creation of preventive measures for the development of tooth decay that leads many children and teens to early loss of teeth. Thus, we sought to evaluate the presence of tooth decay and food habits in schoolchildren of 10 to 14 years of a municipal school in the city of Picos, Piaui. Cross-sectional observational study made with 69 students regular enrolled and in the range of 10-14 years. Questionnaires were applied with questions regarding oral hygiene, use of dental services and eating habits with an emphasis on sugar consumption. Clinical examination for detecting carious lesions were also conducted. The descriptive analysis of the data was represented through tables and graphs. In $66.6 \%$ of subjects decay was present, and $7.4 \%$ of them had six or more lesions. Cereals, cookies and soft drinks were some of the food more consumed $(78.2 \%, 60.8 \%$ and $55 \%$ respectively) and only $44.9 \%$ of students reported having brushing after snacks. It is concluded that in the study population, most individuals had teeth with lesions of dental caries and a diet rich in carbohydrates and sugars.
\end{abstract}

Keywords: Food Habits. Nutrition, Public Health. Dental Caries. Oral Hygiene.

\section{Introdução}

A dieta e o estado nutricional influenciam a saúde bucal de diversas formas. Deficiências no estado nutricional podem resultar em defeitos na estrutura dos dentes durante o seu período de formação e atraso na erupção dentária, além de alterações nas glândulas salivares. Após a erupção, por um efeito local direto, os açúcares e ácidos da dieta podem causar cárie dentária e erosão dentária respectivamente ${ }^{1}$.

Muitos estudos têm procurado verificar a influência de uma alimentação saudável com a formação e o desenvolvimento dos dentes, pois de acordo com Lessard ${ }^{2}$ a alimentação e a nutrição, por meio de uma série de relações complexas, são importantes no desenvolvimento dentário, assim como o perfil alimentar é importante no aparecimento da cárie e um fator primário de determinação da susceptibilidade para a doença. Segundo Tomita et al. ${ }^{3}$, a cárie dentária é uma doença infectocontagiosa multifatorial, resultante de várias reações químicas complexas com formação de ácidos que causam a destruição do esmalte dentário e posteriormente, se não tratada, pode resultar na perda completa do dente ${ }^{4}$.

De acordo com Bezerra e Toledo ${ }^{5}$, a cárie dentária decorre da dinâmica entre biofilme dental, carboidratos e saliva, determinando o seu conceito como doença infecciosa bacteriana, modificada por uma dieta rica em carboidratos, induzindo à formação de biofilme com microrganismos cariogênicos, como os Streptococcus mutans, com a formação de ácidos orgânicos que reduzem drasticamente o $\mathrm{pH}$ do meio bucal, promovendo a subsaturação de cálcio e fósforo com consequente desmineralização das estruturas dentárias pela perda de íons de hidroxiapatita.

Outro aspecto importante na etiologia da cárie dentária é 
a frequência do consumo de açúcar, devido ao processo de mudança nos hábitos alimentares do homem com aumento na preferência por alimentos açucarados em substituição aos alimentos naturais. Essa alteração tem sido acompanhada por elevação na incidência da doença ${ }^{3}$ e que, segundo autores como Walter et al. ${ }^{6}$, apesar da necessidade da participação de carboidratos fermentáveis para o surgimento de lesões cariosas, o fator dieta deve ser analisado com base no conceito de multicausalidade das doenças, pois não existe nenhum alimento capaz de causar cárie sem a interação de outras variantes biológicas e não biológicas neste processo.

Nos estudos conduzidos por Kramer, Feldens e Romano ${ }^{7}$ e Stecksen-Blicks, Holm ${ }^{8}$ uma alimentação adequada com orientação nutricional e correta escovação podem desempenhar papel importante no controle da cárie, tornandose fatores positivos para o não aparecimento da doença. Ao contrário, crianças com alta ingestão de lanches e frequência de escovação irregular têm elevada incidência de lesões cariosas.

Observações realizadas por Shils et al. ${ }^{9}$ sugerem que a dieta e a nutrição estão intimamente relacionadas à saúde geral, oral e à prevenção de doenças, de forma que a saúde bucal exerce grande influência sobre a dieta e o estado nutricional, assim como a dieta pode afetar os tecidos orais direta ou indiretamente.

Dessa forma, sabendo-se que a dieta do adolescente constitui fator importante na etiologia e progressão da doença cárie e, que, a discriminação quanto à preferência por sabores ocorre com o desenvolvimento da criança e é afetada por inúmeros fatores, é fundamental a orientação não só quanto aos hábitos adequados sobre alimentação e higiene bucal, como também em relação ao consumo racional de açúcar, visando a promoção de saúde bucal.

Portanto, dieta, nutrição, saúde geral e bucal estão interligadas, e uma dieta balanceada acarreta um bom estado nutricional que contribui para uma boa saúde oral e geral dos indivíduos. Hábitos alimentares sadios devem ser estabelecidos, dando preferência à ingestão de uma alimentação balanceada e saudável, incluindo vegetais, frutas e verduras, no intuito de manter a boca livre de infecções e doenças, fatores importantes para a determinação e conservação da saúde bucal e geral dos indivíduos. Dessa forma, o objetivo do presente estudo foi avaliar a presença de cárie dentária e os hábitos alimentares de escolares de 10 a 14 anos de uma escola municipal no município de Picos-Piauí.

\section{Material e Métodos}

A presente pesquisa foi submetida e aprovada pelo Comitê de Ética da Universidade Federal do Piauí com o certificado de número 0048.0.045.000-10. Aos pais foi solicitada a assinatura do Termo de Consentimento Livre e Esclarecido - TCLE para que seus filhos participassem da pesquisa, de acordo com as diretrizes da resolução 466/12 do Conselho Nacional de Saúde.
Trata-se de um estudo observacional transversal no qual a população foi constituída por indivíduos na faixa etária de 10 a 14 anos, regularmente matriculados na escola municipal Urbano Maria Eulálio, na cidade de Picos, Piauí, Brasil. A amostra foi do tipo censitária, composta de 69 escolares, ou seja, todas as crianças matriculadas na escola e com a faixa etária selecionada foram incluídas. Os critérios de exclusão foram a ausência nos dias de visita e indivíduos com deficiências neurológicas.

Inicialmente, foi aplicado aos alunos um questionário estruturado com perguntas abertas e fechadas que abordaram temas referentes à higiene bucal, utilização de serviços odontológicos e hábitos alimentares com ênfase no consumo de açúcar. Em visita posterior à escola foram realizados exames clínicos com auxílio de espátula de madeira (abaixador de língua) e espelho bucal plano número 5 sob luz natural, visando à identificação de elementos dentários com lesões cariosas. Todos os procedimentos foram realizados por um mesmo examinador, previamente treinado por um profissional competente da área.

Os alunos foram divididos em cinco grupos de acordo com a faixa etária (10, 11, 12, 13 e 14 anos). Foram entregues Kits contendo escova e dentifrício fluoretado para realização de higiene bucal prévia aos exames clínicos. A presença de cárie inicial (lesões de mancha branca), lesões cariosas cavitadas e necessidade de exodontia foram registradas em filhas odontológicas convencionais. Ao final da coleta de dados, os alunos participaram de atividades educativas e palestras ministradas pelo examinador, com objetivo de orientá-los com relação à cárie, seus fatores etiológicos, agravantes e formas de prevenção. A análise dos dados foi realizada por meio do programa estatístico SPSS versão 18.0. A análise descritiva dos dados foi representada em tabelas e gráficos.

\section{Resultados e Discussão}

Para Batista et $a l .^{10}$, uma alimentação saudável iniciada ainda na infância contribui para crescimento e desenvolvimento adequados, prevenção de doenças ${ }^{11}$ e saúde bucal, refletindo assim na qualidade de vida geral do indivíduo. Uma alimentação saudável é aquela que oferece, em todos os ciclos de vida de uma pessoa, os nutrientes necessários para o seu bem-estar e saúde, indo além do simples fornecimento de nutrientes ${ }^{9,12}$.

Apesar do declínio nos níveis de cárie dentária pelo mundo, o fenômeno de polarização da doença, ou seja, a concentração de altos índices de cárie em pequenos grupos populacionais de uma mesma região tem aumentado a discussão quanto aos fatores sociais e comportamentais associados à ela ${ }^{13}$. O consumo elevado de alimentos ricos em açúcar é considerado fator de risco para várias doenças dentre elas a cárie; contudo, uma dieta balanceada em contrapartida concorre positivamente para a prevenção da doença, fornecendo nutrientes e evitando a instalação das bactérias cariogênicas ${ }^{11}$.

No Brasil, a cárie ainda se constitui um problema de saúde 
pública. Segundo os dados do Ministério da Saúde ${ }^{14}, 53,4 \%$ das crianças com cinco anos possuem cárie, aos 12 anos este percentual se eleva para $56,5 \%$ e na faixa etária entre 15 e 19 anos chega a 76,1\%. Portanto, estudos sobre fatores diretamente relacionados à cárie dental, como a dieta, são sempre atuais e necessários.

Neste estudo os alimentos mais consumidos foram carnes (84\%), seguido de cereais como pão, arroz e macarrão (78,2\%), biscoitos recheados, refrigerantes e frituras $(60,8 \%, 55 \%$, $46,3 \%)$. Os legumes $(31,8 \%)$ foram os menos consumidos, conforme observado no Quadro 1. Palma, Escrivão e Oliveira $^{15}$ e Gueiros, Silva ${ }^{16}$ relataram que alimentos ricos em amido são preditivos para a cárie dentária, pois este componente promove a formação de placas acidogênicas após sua hidrólise. No estudo de Bonotto et al. ${ }^{17}$ avaliou-se a prevalência de cárie dentária em escolares na faixa etária de 12 anos. Os resultados mostraram que 37,9\% dos meninos e $39,7 \%$ das meninas consumiam mais de seis alimentos com sacarose por dia. Porém, a prevalência de cárie foi 48,2\% e $43,4 \%$ respectivamente, em razão da frequência de escovação ser maior ou igual a duas vezes ao dia em $36,8 \%$ dos meninos e $47,2 \%$ das meninas.

Quadro 1: Alimentos consumidos pelos adolescentes. PicosPiauí, 2011

\begin{tabular}{|c|c|c|}
\hline Alimentos & $\begin{array}{c}\mathbf{n}^{\mathbf{0}} \text { de Adolescentes que } \\
\text { Consomem cada Alimento }\end{array}$ & $\%$ \\
\hline Carnes & 58 & 84 \\
\hline Cereais & 54 & 78,2 \\
\hline Sucos & 51 & 73,9 \\
\hline Frutas & 48 & 69,5 \\
\hline Leite & 45 & 65,2 \\
\hline Ovos & 45 & 65,2 \\
\hline Biscoito Recheado & 42 & 60,8 \\
\hline Refrigerantes & 38 & 55 \\
\hline Frituras & 32 & 46,3 \\
\hline Verduras & 32 & 46,3 \\
\hline Doces & 32 & 46,3 \\
\hline Salgadinhos & 30 & 43,4 \\
\hline Legumes & 22 & 31,8 \\
\hline
\end{tabular}

Fonte: Dados da pesquisa.

Nos participantes da presente pesquisa foi observada alta prevalência de elementos dentários com lesões cariosas $(66,6 \%)$, principalmente em pré-molares e molares. Fato associado principalmente à morfologia destes dentes, que facilita maior acúmulo de resíduos alimentares e dificulta o acesso à escovação (Quadro 2).

Quadro 2: Dados sobre a experiência de cárie da amostra estudada. Picos-Piauí, 2011

\begin{tabular}{|c|c|c|}
\hline Número de alunos & Número de dentes cariados & $\mathbf{\%}$ \\
\hline 23 & Todos os dentes hígidos & 33,4 \\
\hline 7 & 1 cárie & 10,1 \\
\hline 11 & 2 cáries & 15,9 \\
\hline 11 & 3 cáries & 15,9 \\
\hline 8 & 4 cáries & 11,5 \\
\hline 4 & 5 cáries & 5,8 \\
\hline 5 & 6 ou mais cáries & 7,4 \\
\hline Total 69 & & $100 \%$ \\
\hline
\end{tabular}

Fonte: Dados da pesquisa.
De acordo com Bernabé et $a l .{ }^{18}$, a cárie dentária é uma doença mediada pela dieta, sendo os açúcares essenciais no processo carioso. Em seu estudo, observou que a quantidade de ingestão de açúcares foi mais relevante para o desenvolvimento da cárie que a frequência. Além disso, o uso diário de creme dental com flúor reduziu, porém não eliminou a associação entre a quantidade de ingestão de açúcares e a cárie dentária. A escovação com dentifrício fluoretado, principalmente após as refeições é uma das principais maneiras de se evitar a cárie dentária $^{19}$. No gráfico da Figura 1 observa-se que muitos dos escolares ainda não realizam a higienização adequada após as refeições.

Figura 1: Frequência de escovação dos adolescentes. PicosPiauí, 2011

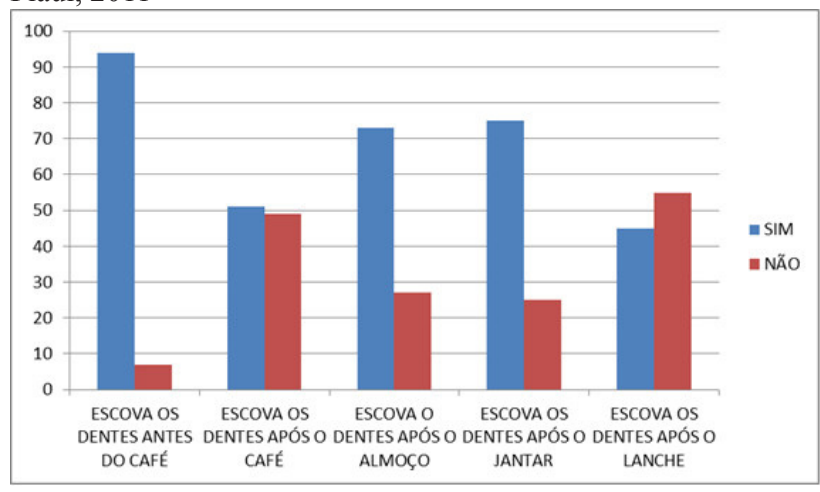

Fonte: Dados da pesquisa.

De acordo com Palma et al..$^{15}$, o uso de dentifrício fluoretado e a fluoretação da água de abastecimento público auxiliam na diminuição da prevalência de cárie. Gonçalves et $a l .{ }^{20}$ investigaram a associação entre experiência de cárie em crianças e a disponibilidade domiciliar de açúcar no Brasil e relataram que a fluoretação explica os níveis de cárie mais baixos. Com o objetivo de se obter informações acerca da fluoretação da água de abastecimento do município de Picos, Piauí uma visita à empresa responsável pelo tratamento e abastecimento de água da cidade foi realizada. Foi relatado que por motivos financeiros a água do município não recebe a devida fluoretação.

Em cinco $(7,4 \%)$ indivíduos foi observada a presença de seis ou mais lesões cariosas. Catorze dentes decíduos possuíam indicação de exodontia por terem lesão em estado avançado, impossibilitando sua restauração e manutenção do dente na cavidade oral. Apenas 21,7\% dos participantes relataram visitar o dentista em intervalos de seis meses, tempo indicado para periodicidade das visitas possibilitando a prevenção, o controle e a manutenção da saúde bucal.

Estudo realizado por Gueiros e Silva ${ }^{16}$ apontou que o consumo de lanches entre as refeições leva à maior prevalência de cárie quando comparado a pessoas que não têm esse hábito. Na presente pesquisa, dentre os escolares que fazem lanches entre as refeições ou antes de dormir, somente 44,9\% realizam a escovação ao final. A maioria faz entre quatro e seis refeições por dia, tornando os lanches tão importantes quanto 
as principais refeições. Dessa forma, para Mahan et al. ${ }^{12}$ os lanches devem ser selecionados de forma cuidadosa para que sejam os mais ricos em nutrientes e menos cariogênicos possíveis.

Punitha et al..$^{21}$ avaliaram o papel dos hábitos alimentares e da dieta na ocorrência e gravidade de cárie dentária em escolares na faixa etária de 13 a 19 anos. Não houve diferença estatisticamente significativa na prevalência de cárie $(36,7 \%)$ quanto à frequência no consumo de fast foods, lanches entre as refeições e dieta vegetariana ou não. A ingestão de bebidas carbonatadas e produtos de confeitaria (doces e chocolates) em contrapartida mostraram associação significativa com a ocorrência e gravidade da cárie. No presente estudo, da mesma forma, muitos escolares relataram fazer consumo de biscoitos recheados, refrigerantes e frituras $(60,8 \%, 55 \%$, $46,3 \%)$, sendo a prevalência de cárie elevada $(66,7 \%)$.

Observou-se que somente $50,7 \%$ dos adolescentes relataram consumir leite no café da manhã. De acordo com Campos e Demonte ${ }^{22}$, a proteína do leite, a caseína, reduz a solubilidade do esmalte dentário ao se ligar a este. $\mathrm{O} \mathrm{pH}$ da placa é aumentado pela ureia, diminuindo a aderência de microrganismos, reforçando a tese de que uma alimentação balanceada e adequada tem papel importante na determinação da saúde bucal e geral dos indivíduos ao longo da vida ${ }^{10}$.

Guerreiros e $\operatorname{Silva}^{23}$ relataram que comportamentos adequados dependem da consciência e de noções básicas de higiene, promovendo saúde e contribuindo para a boa qualidade de vida dos indivíduos. Segundo Navarro e Cortes ${ }^{24}$ e Stelluto $\mathrm{Jr}^{25}$ o papel preventivo da mãe com relação ao desenvolvimento de lesões cariosas em seus filhos é de fundamental importância. A implementação da educação em saúde bucal e nutrição, por meio de programas preventivos, pode estabelecer hábitos alimentares saudáveis reduzindo assim a incidência de cárie e favorecendo a saúde geral ${ }^{26}$.

A obesidade, as doenças dela decorrentes e a cárie dentária têm no hábito alimentar um componente etiológico comum, portanto o aconselhamento dietético é fundamental para a saúde bucal ${ }^{27}$. Hábitos alimentares adquiridos na infância formam a base para o futuro padrão alimentar do indivíduo. No entanto, deve-se sempre levar em consideração, a realidade em que os indivíduos vivem, tendo como objetivo central a utilização racional do açúcar ${ }^{6,28-30}$.

\section{Conclusão}

Na população estudada, a maioria dos indivíduos tinha elementos dentários com lesões cariosas e alimentação rica em carboidrato e açúcares. Sugere-se que medidas informativas com o objetivo da prevenção devem ser realizadas a fim de introduzir hábitos alimentares saudáveis, auxiliando dessa forma na manutenção da saúde bucal e geral.

\section{Referências}

1. Freire MCM, Balbo PL, Amador MA, Sardinha LMV. Guias alimentares para a população brasileira: implicações para a Política Nacional de Saúde Bucal. Cad Saude Publica 2012;28:20-9.

2. Lessard GM. Discussion: nutritional aspects of oral healthnew perspectives. Am J Clin Nutr 1995;61(2):446S.

3. Tomita NE, Bijela VT, Lopes ES, Franco LJ. Prevalência de cárie dentária em crianças de faixa etária de 0 a 6 anos matriculada em creches: importância dos fatores socioeconômicos. Rev Saude Publica 1996;30(5):413-20.

4. Guedes-Pinto AC. Odontopediatria. São Paulo: Santos; 1995.

5. Bezerra ACB, Toledo OAT. Nutrição, dieta e cárie. In: Kriger L. Promoção de saúde bucal - ABOPREV. São Paulo: Artes Médicas; 1999.

6. Walter LRF, Ferelle A, Issao M. Dieta e cárie na primeira infância: odontologia para o bebê. São Paulo: Artes Médicas; 1997.

7. Kramer PF, Feldens CA, Romano AR. Promoção de saúde bucal em odontopediatria: diagnóstico, prevenção e tratamento da cárie oclusal. São Paulo: Artes Médicas; 1997.

8. Stecksén-Blicks C, Holm AK. Between- meal eating, toothbrushing frequency and dental caries in 4-yearold children in the north of Sweden. Int J Paediatr Dent 1995;5(2):67-72.

9. Shils ME, Shike M, Ross AC, Cousins RJ, Caballero B. Nutrição moderna na saúde e na doença. Barueri: Manole; 2003.

10. Batista LRV, Moreira EAM, Corso ACT. Alimentação, estado nutricional e condição bucal da criança. Rev Nutr 2007;20(2):191-6.

11. Fadel CB. Cárie dental precoce: qual o verdadeiro impacto da dieta em sua etiologia? Publ UEPG Cienc Biol Saúde 2003;9(3/4):83-9.

12. Mahan LK, Escott-Stump S, Raymond JL. Krause: alimentos, nutrição e dietoterapia. Rio de Janeiro: Elsevier; 2012.

13. Peres KGA, Bastos JRM, Latorre MRDO. Severidade de cárie em crianças e relação com aspectos sociais e comportamentais. Rev Saúde Pública 2000;34(4):402-8.

14. Brasil. Ministério da Saúde. Secretaria de Atenção à Saúde. Secretaria de Vigilância em Saúde. SB Brasil 2010: Pesquisa Nacional de Saúde Bucal: resultados principais. Brasília: MS; 2012.

15. Palma D, Escrivão MAMS, Oliveira FLC. Guia de nutrição na infância e na adolescência. Barueri: Manole; 2009.

16. Gueiros LAM, Silva MDP. Inquérito de Cárie dental e perfil alimentar em escolares de 6 a 12 anos de duas populações da Região Metropolitana de Recife. Odontol Clin Cientif 2003;2(3):201-9.

17. Bonotto DMV, Pintarelli TP, Santin G, Montes GR, Ferreira FM, Fraiz FC. Cárie dentária e gênero em adolescentes. RFO 2015;20(2): 202-7.

18. Bernabé E, Vehkalahti MM, Sheiham A, Lundqvist A, Suominen AL. The shape of the dose-response relationship between sugars and caries in adult. J Dent Res 2015. doi:10.1038/sj.bdj.2015.951

19. Ribeiro NME, Ribeiro MAS. Aleitamento materno e cárie do lactente e do pré- escolar: uma revisão crítica. Ed J Pediatr 2004;80(5):199-210.

20. Gonçalves MM, Leles CR, Freire MCM. Cárie dentária, disponibilidade de açúcar e fatores associados na capitais brasileiras em 2003: um estudo ecológico. Rev Odontol 
2012;41(6):425-32.

21. Punitha VC, Amudhan A, Sivaprakasam P, Rathanaprabu V. Role of dietary habits and diet in caries occurrence and severity among urban adolescent school children. J Pharm Bioallied Sci 2015;7:S296-300.

22. Campos JADB, Demonte A. Interferência dos nutrientes nas doenças bucais de caráter de saúde pública. Rev Fac Odontol Lins 2003;15(1):53-7.

23. Guerreiros LAM, Silva MDP. Inquérito de cárie dental e perfil alimentar em escolares de 6 a 12 ano de duas populações da Região Metropolitana do Recife. Odontol Clin Cientif 2003;2(3):201-10.

24. Navarro MFL, Cortes DF. Avaliação e tratamento do paciente com relação ao risco de cárie. Maxiodonto 1995;1(4):18-23.

25. Stelluto Jr A. O primeiro vício. Rev ABO Nac 1995;3(1):6-8.

26. Dias ACG, Raslan S, Scherma AP. Aspectos nutricionais relacionados à prevenção de cáries na infância. ClipeOdonto 2011;3(1):37-44.

27. Traebert J, Moreira EAM, Bosco VL, Almeida ICS. Transição alimentar: problema comum à obesidade e à cárie dentária. Rev Nutr 2004;17(2):247-53.

28. Tashima AY, AP Verrastro, SLM Ferreira. Correlação entre o aconselhamento dietético-nutricional e a promoção de saúde na clínica de odontopediatria. J Bras Odontoped Odontol Bebê 2000;3(16):505-12.

29. Campos JADB, Zuanon ACC, Campos AG. Influência da alimentação e da nutrição na odontogênese e desenvolvimento de lesões de cárie dental. JBP J Bras Odontopediatr Odontol Bebê 2003;6(31):246-9.

30. Chaves AMB, Rosenblatt A, Colares V. A importância da dieta do desmame na saúde oral. JBP J Bras Odontopediatr Odontol Bebê 2003;6(30):158-62. 